\title{
CARACTERIZACIÓN HISTOPATOLÓGICA DE LESIONES BENIGNAS DE LA MAMA EN MUJERES DEL DEPARTAMENTO DE SUCRE, COLOMBIA. PERIODO 2012-2013
}

\author{
HISTOPATHOLOGICAL CHARACTERIZATION OF BENIGN BREAST LESIONS IN \\ WOMEN OF THE DEPARTMENT OF SUCRE, COLOMBIA. PERIOD 2012-2013
}

\author{
EDGAR VERGARA DAGOBETH, ${ }^{1}$ Med. Esp, KATTY LACAYO LEPESQUEUR, ${ }^{2}$ Med. Esp; \\ YEIMER ORTIZ MARTÍNEZ, ${ }^{3}$ Est, MARTÍNEZ TORRES, ANDONYS ${ }^{3}$ Est.
}

${ }^{1}$ Profesor asociado de Cirugía, Universidad de Sucre. Sincelejo-Colombia. $2^{*}$ Profesor Asistente de Patología, Universidad de Sucre. Sincelejo-Colombia. ${ }^{3}$ Estudiantes de Medicina, Universidad de Sucre. Sincelejo-Colombia.

\section{Key words:}

Benign breast disease, fibroadenoma, proliferative lesion, atypia,

breast cancer.

\section{Palabras Clave:}

Enfermedad benigna de mama, fibroadenoma, lesiones proliferativas, atipia, cáncer de mama
INFORMACIÓN

Recibido: 15-04-2015;

Aceptado: 15-09-2015.

Correspondencia autor:

kattylacayo7@yahoo.com

\begin{abstract}
The present study re-classify the histopathological pattern of benign breast lesions in women of the Department of Sucre, in the period 2012-2013. Cross-sectional descriptive-retrospective in patients with benign pathology report breast lesion study, assisted in the pathology laboratory Associated Pathologists of Sucre Ltda, Sincelejo-Sucre. Plates, file cases of benign breast disease, were reviewed by a pathologist. The diagnosis was reconfirmed and was reclassified to the classification of Page and Dupont and no proliferative lesion was found in 376 (82.27\%), proliferative lesion without atypia in $67(14.66 \%)$ and proliferative lesion with atypia $14(3.06 \%)$. No proliferative lesions are most frequent in the department of Sucre and the most common benign breast lesion in this study was the fibroadenoma. Pathology reports should be reported according to the classification of Page and Dupont, to improve prediction and risk stratification and better risk-reduction counseling for women with atypical hyperplasia. Monitoring should be performed with routine mammographic screening in these high risk groups.
\end{abstract}

\section{Resumen}

El presente trabajo reclasificar el patrón histopatológico de las lesiones benignas de mama en mujeres del departamento de Sucre, en el período 2012-2013. Se trata de un estudio observacional-descriptivo-transversal, retrospectivo, en pacientes con reporte de patología de lesión benigna de mama, atendidas en el laboratorio de patología Patólogos Asociados de Sucre Ltda, Sincelejo-Sucre. Se revisaron las placas de archivos de casos de patología benigna de mama. Se reconfirmó el diagnóstico y se reclasificó de acuerdo con Page y Dupont y se encontró lesión no proliferativa en $376(82,27 \%)$, lesión proliferativa sin atipias en $67(14,66 \%)$ y lesión proliferativa con atipias en $14(3,06 \%)$. Las lesiones no proliferativas son las lesiones benignas de mama más frecuentes en el departamento de Sucre y la más común en este estudio fue el fibroadenoma. Los informes de patología se deben reportar según la clasificación de Page y Dupont, para la mejora de la predicción y estratificación de riesgos y el mejor asesoramiento de reducción de riesgo para las mujeres con hiperplasia atípica. Se debe realizar seguimiento con mamografías preventivas de rutina en estos grupos de alto riesgo. 
Introducción

Las enfermedades benignas de mama constituyen un grupo heterogéneo de trastornos que incluyen anormalidades del desarrollo, proliferaciones epiteliales y estromales, lesiones inflamatorias y neoplasias con una amplia gama de características histopatológicas (TAVASSOLI, 2003). Las investigaciones epidemiológicas indican que más del $50 \%$ de todas las mujeres tendrán alguna enfermedad benigna de mama después de los 20 años (GOEHRING, 1997). Desde hace tiempo se ha reconocido que algunas lesiones benignas de mama eventualmente pueden progresar a cáncer de mama (DUPONT et al., 1994; SINGLETARY, 2003). Dupont y Page examinaron 10.366 biopsias de mama y clasificaron las lesiones benignas de mama en tres categorías: lesiones no proliferativas, lesiones proliferativas sin atipia y lesiones proliferativas con atipia. El riesgo de cáncer de mama se ha asociado con características histopatológicas específicas de lesiones benignas de mama; existiendo un mayor riesgo en las lesiones proliferativas. (DUPONT y PAGE, 1985; FITZGIBBONS et al., 1998; WANG et al., 2008; WORSHAM et al., 2007). Así, en las pacientes con lesiones no proliferativas el riesgo de desarrollar cáncer de mama es el mismo que las pacientes de su misma edad que no padecen ninguna alteración; las que tienen lesiones proliferativas sin atipias presentan un riesgo relativo de 1.5-2, y por último, las pacientes con diagnóstico histológico de lesión proliferativa con atipias presentan una elevación del riesgo de cáncer de mama de 4-5 veces (DUPONT y PAGE, 1985). En consecuencia, existe un creciente interés en la enfermedad benigna de mama por su prevalencia y el riesgo de desarrollar cáncer de mama. (CHENG et al., 2008).

En el departamento de Sucre es frecuente la patología benigna de mama. Pero, en el laboratorio de patología, donde se procesan el $95 \%$ de éstas, no utiliza la clasificación de Page y Dupont, la más utilizada para orientar el riesgo de cáncer de mama. Surge así la inquietud de: ¿Cuál es el patrón histopatológico prevalente de las lesiones benignas de mama en el Departamento de Sucre?

La importancia de conocer esta información se traduce en la realización de un mejor seguimiento médico, de las pacientes con lesiones benignas de la mama, que tengan un patrón histopatológico proliferativo; para poder fortalecer los programas de detección temprana en mujeres en riesgo de cáncer de mama.

\section{Materiales y método}

Se realizó un estudio observacional-descriptivotransversal de carácter retrospectivo (MARRETOS et al., 2013), en pacientes con reporte de patología de lesión benigna de mama, atendidas en el laboratorio de patología Patólogos Asociados de Sucre Ltda., de Sincelejo-Sucre; en el periodo comprendido entre el 1 de enero 2012 al 31 de diciembre 2013. El objetivo del estudio fue reevaluar y clasificar el patrón histopatológico de las lesiones benignas de mama en mujeres del departamento de Sucre, en el período 20122013. Las variables incluidas fueron edad, procedencia y tipo histopatológico de la lesión benigna.

Se utilizó un sistema informático diseñado para obtener la información acerca de los datos de cada paciente. Luego de recolectar todos los datos y códigos diagnósticos de las pacientes se procedió a la búsqueda de las diferentes placas histopatológicas con las que contaba cada uno de los casos clínicos registrados en el sistema, éstas se encontraban en la sección de archivos del laboratorio.

Las placas histológicas almacenadas de cada paciente fueron evaluadas por un patólogo. Se reconfirmó el diagnóstico y se reclasificó de acuerdo a la clasificación de Page y Dupont; en las siguientes categorías:

1. Lesiones Benignas no proliferativa: Adenosis microglandular, metaplasia apocrina, quistes simples, ectasia ductal, alteración fibroquística y fibroadenoma.

2. Lesiones Benignas proliferativas sin atipia: Hiperplasia ductal moderada o florida, adenosis esclerosante florida y el papiloma intraductal.

3. Lesiones proliferativas con atipia: Hiperplasia ductal atípica e hiperplasia lobulillar atípica

Se hizo la distribución por edad de la Enfermedad benigna de mama de acuerdo a los rangos de edad de la clasificación ANDI: Aberrations of Normal Development and Involution.

Se tuvo en cuenta la asociación entre el hallazgo histopatológico y el riesgo de cáncer de mama, la edad al momento del diagnóstico de la enfermedad benigna de mama. Los principales efectos de cada variable y los términos de interacción correspondientes se incluyeron en cada figura, y la significación estadística de cada interacción se evaluó de acuerdo a los resultados. Todos los datos fueron introducidos y analizados mediante Epi Info 7 (versión libre). 


\section{Resultados}

Se encontraron 457 reportes patológicos de mujeres con enfermedad benigna de la mama; 298 (65\%) procedían del área urbana del departamento de Sucre. Las edades oscilaron entre 13 a 87 años, con una media de 37 años. El $43,7 \%$ de las pacientes eran menores de 35 años (Figs. 1-4).

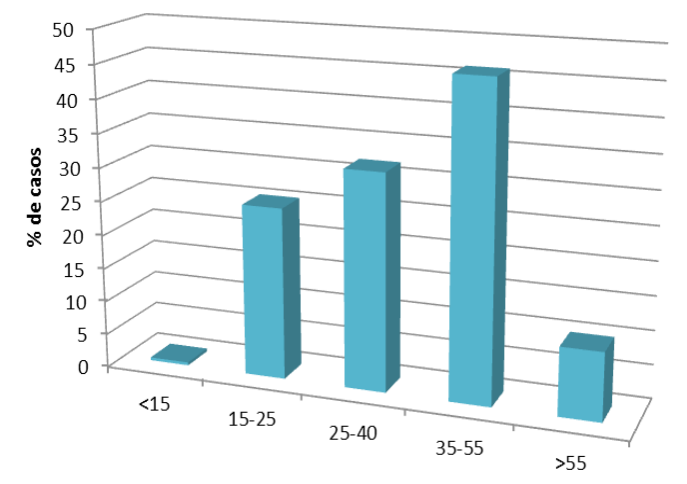

Figura 1. Distribución por edad de la Enfermedad Benigna de Mama en las pacientes del departamento de Sucre.

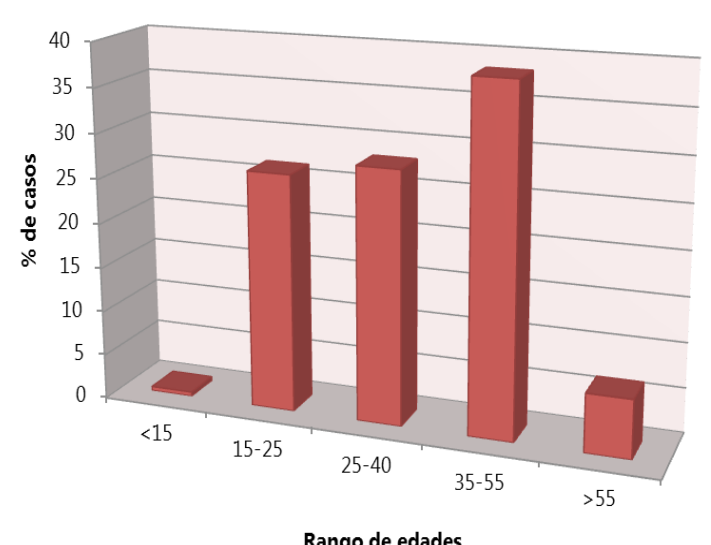

$\overline{\text { Figura 2. Distribución por edad de las lesiones no proliferativas }}$ de mama en las pacientes del departamento de Sucre.

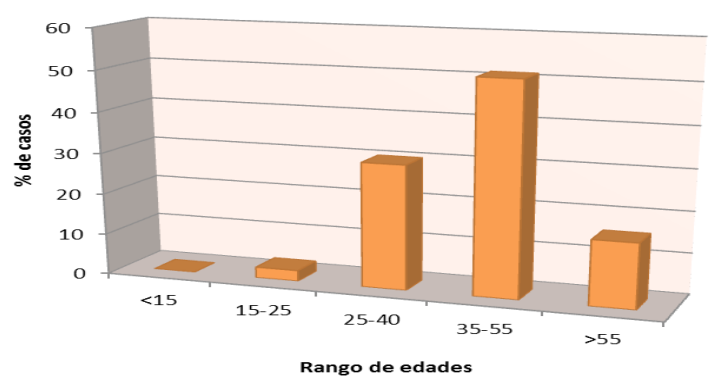

Figura 3. Distribución por edad de las lesiones proliferativas de mama en las pacientes del departamento de Sucre.

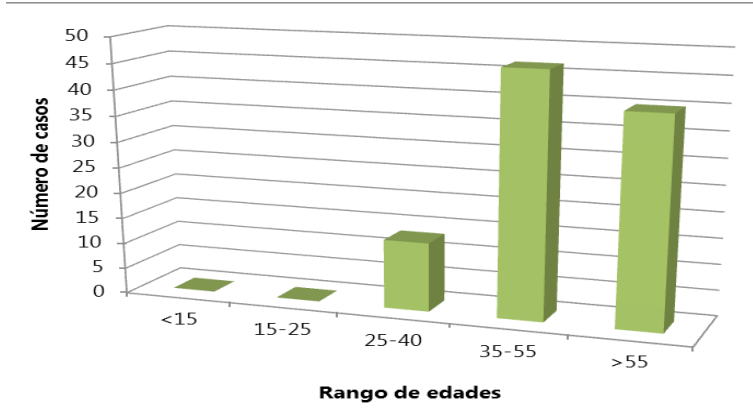

Figura 4. Distribución por edad de las lesiones proliferativas con atipias de mama en el departamento de Sucre

De los casos estudiados, $312(68,7 \%)$ tenían el diagnóstico histopatológico de lesión benigna de mama especificado y $145(31,73 \%)$ no tenían especificado el tipo de lesión benigna.

Al reevaluar los casos de lesión benigna especificada se encontró que, en $202(44,2 \%)$ el diagnóstico histopatológico inicial se reconfirmó. Y en 110 (24,07\%) casos el diagnosticó histopatológico varió. Así, de las 38 muestras diagnosticadas inicialmente como "Lesión quística benigna”, se reconfirmó, quiste macro/micro en 28; mientras que en 10 varió a alteración fibroquística. De las 22 muestras diagnosticadas inicialmente como "Mastopatía no proliferativa", se reconfirmó, alteración fibroquística en 17, adenosis microglandular en 3; mientras que en $2(0,437 \%)$ varió a hiperplasia ductal florida. De las 50 muestras diagnosticadas inicialmente como "Hiperplasia epitelial", se reconfirmó, hiperplasia ductal florida en 36; mientras que en 14 (3,063\%) se encontró cambios atípicos (hiperplasia ductal atípica en 13 casos e hiperplasia lobulillar atípica en 1 caso). De los $145(31,73 \%)$ casos que tenían el diagnóstico histopatológico de "lesión benigna de mama" sin otra especificación, al reevaluarlos se encontró que correspondían a: fibroadenoma en 92, alteración fibroquística en 43 y quiste simple macro/micro en 10 casos.

En general, el fibroadenoma se diagnosticó en 183 $(40 \%)$ de casos, seguido por la alteración fibroquística con $103(22,5 \%)$ de casos.

Al distribuir los reportes de patología de las lesiones benignas de mama, de acuerdo a la clasificación de Page y Dupont, se encontró lesión no proliferativa en $376(82,27 \%)$, lesión proliferativa sin atipias en 67 $(14,66 \%)$ y lesión proliferativa con atipias en $14(3,06 \%)$ (Tabla 1). 
Tabla 1. Clasificación histopatológicas de las lesiones benignas de mama en el Departamento de Sucre. Período 2012-2013.

\begin{tabular}{|c|c|c|}
\hline \multicolumn{3}{|c|}{ Lesiones no proliferativas } \\
\hline Tipo lesión & Cantidad & Porcentaje (\%) \\
\hline Adenosis & 5 & 1,09 \\
\hline Metaplasia apocrina & 6 & 1,31 \\
\hline Quistes macro/micro & 41 & 8,97 \\
\hline Ectasia ductal & 38 & 8,32 \\
\hline Alteración fibroquística & 103 & 22,5 \\
\hline Fibroadenoma & 183 & 40 \\
\hline Subtotal & 376 & 82,27 \\
\hline \multicolumn{3}{|c|}{ Lesiones proliferativas epiteliales sin atipia } \\
\hline Hiperplasia ductal moderada o florida & 42 & $9,19 \%$ \\
\hline Adenosis esclerosante florida & 12 & $2,63 \%$ \\
\hline Papiloma intraductal & 13 & $2,84 \%$ \\
\hline Subtotal & 67 & 14,66 \\
\hline \multicolumn{3}{|c|}{ Lesiones proliferativas epiteliales con atipias } \\
\hline Hiperplasia ductal atípica & 13 & $2,84 \%$ \\
\hline Hiperplasia lobulillar atípica & 1 & $0,22 \%$ \\
\hline Subtotal & 14 & 3,06 \\
\hline
\end{tabular}

El rango de edad más afectado por enfermedad benigna de mama fue el de 35-55 años con 45\%. Al discriminarla por tipo de lesión patológica, las no proliferativas se presentaron en menores de 40 años un 57\%; las proliferativas sin atipias en mayores de 40 años un 64\%; y las proliferativas con atipias en mayores de 40 años con un $86 \%$ (Figs. 2-4).

\section{Discusión}

Se encontró que la patología benigna de mama se compone con mayor frecuencia de lesiones no proliferativas $(82,27 \%)$, seguido por las lesiones proliferativas sin atipia $(14,66 \%)$, y con menos frecuencia, las lesiones proliferativas atípicas (3,06\%). Resultados que siguen la tendencia del estudio realizado por (SCHNITT et al., 1993) con 266 biopsias de mujeres con enfermedad benigna de mama de Japón, donde las lesiones no proliferativas representaron el $81,6 \%$, las lesiones proliferativas sin atipia el $16,2 \%$; y las lesiones proliferativas con atipia el $2 \%$. También una investigación realizada por (CHENG et al., 2008) con 4.970 mujeres con diagnóstico por biopsias de enfermedad benigna de mama en el Sistema de Salud Henry Ford en EEUU, muestra que las lesiones no proliferativas representaron el $62,5 \%$, las lesiones proliferativas sin atipia el $34.7 \%$; y las lesiones proliferativas con atipia el $2,4 \%$.
El fibroadenoma fue la enfermedad benigna de mama más común en este estudio representando el 40,04\% de los casos. Este fue también el hallazgo en la mayoría de los estudios previos realizados en Perú, Nigeria, Yemen y República Dominicana, donde el fibroadenoma representa el $34,8 \%, 43 \%, 40,5 \%$ y el $37 \%$ de los casos de enfermedad benigna de mama respectivamente.(MARREROS et al., 2013; OLUEDDO et al., 2011; BAFAKEER et al., 2010; GOMERA et al., 2000). También fue más común en Jamaica e Irak, con un $39,4 \%$ y $61,4 \%$ de todas las lesiones benignas de mama respectivamente (SHIRLEY SE, et al., 2008; JABBO et al., 2010). Sin embargo, en un estudio realizado (BOJANINI y VILLEGAS, 1993) en el Hospital General de Medellín, el fibroadenoma fue documentado como el segundo diagnóstico más común, que comprende el $21,9 \%$ de los casos, un resultado que concuerda con los informes procedentes de Pakistán y EEUU, donde el fibroadenoma representó el 29,4\% y el $17,5 \%$, respectivamente (MEMON et al., 2007; TICE et al., 2013).

El fibroadenoma se encontró más frecuente en el rango de edad de $15-25$ años con el $48,02 \%$ de los casos. Acorde a los resultados de un estudio prospectivo realizado durante el período de noviembre 2003 a mayo 2010, con 210 pacientes que asistieron a la consulta externa de cirugía del Hospital Victoria en India mostró un número máximo de casos $(66,1 \%)$ en el rango de edad 15-30 años (EL-WAKEEL et al., 2003) y a un estudio anatomopatológico de enfermedad benigna en Tokio y Nueva York, que mostró el pico más alto de incidencia entre los 15 y 20 años en la población estadounidense y japonesa (SCHUERCH et al., 1982). El desarrollo de los fibroadenomas es hormono-dependiente y generalmente se forman durante la menarquia (15 -25 años de edad), tiempo en el que se añaden estructuras lobulares al sistema ductal de la mama (HUGHES et al., 1987). La incidencia rápidamente disminuye con el incremento en la edad de las pacientes y no se desarrolla o crece después de la menopausia.

La alteración fibroquística, fue el segundo diagnóstico más común en este estudio, representó el $22,5 \%$ de los casos, una cifra consistente con el 23,8\%, $16 \%$ y $19,3 \%$ documentado en Nigeria, Yemen y Jamaica respectivamente (OLU-EDDO et al., 2011; BAFAKEER et al., 2010; GOMERA et al., 2000). En Italia, Medellín y en otros estudios en EE.UU. y Perú, la alteración fibroquística fue la enfermedad benigna de mama más común con el $43,2 \%, 51,6 \%, 47 \%$ y $42 \%$ de los casos respectivamente (BOJANINI y VILLEGAS, 1993; CIATTO et al., 1998; DONEGAN et al., 1995; VALDIVIESO et al., 1999). El rango de edad predominante de las pacientes con alteración fibroquística en este estudio fue de 35 a 55 años, con 
una edad media de 42 años, así como se documenta en varios estudios (HUGHES et al., 1987; OCHICHA et al., 2002).

La ectasia ductal representó el $8,32 \%$ de todas las lesiones benignas de mama en este estudio, similares resultados se encontraron en Perú e Irak, donde representó el 9 y $8,78 \%$ respectivamente (MARREROS et al., 2013; JABBO et al., 2010). Esta entidad es importante porque en algún momento puede presentarse con características clínicas de la enfermedad maligna de mama y mostrar microcalcificaciones en la mamografía, aunque no hay un aumento del riesgo de cáncer de mama (MERIH et al., 2006).

La adenosis esclerosante significó el $18 \%$ de las lesiones proliferativas sin atipia y el $2,63 \%$ de todas las lesiones benignas de mama, relativamente inferior al $9 \%$ reportado en Perú (MARREROS et al., 2013). Es importante reconocer la adenosis esclerosante porque Jensen y colaboradores han demostrado que los casos bien definidos de adenosis esclerosante pueden estar asociados con 1,7 veces mayor riesgo para el desarrollo de cáncer invasivo (WEIDNER, 2003).

En este estudio, el papiloma intraductal se presentó en el $2,84 \%$ del total de pacientes, cifras inferiores al $10 \%$ y $5 \%$ reportados en Tokio y New York, respectivamente (HUGHES et al., 1987). Cuando se excluye la hiperplasia atípica, un papiloma solitario conlleva un riesgo relativo de 2,4 a 2,1 en comparación con 3,01 a 3,54 con micro-papilomas. Cuando la atipia se asocia con el papiloma, este riesgo aumenta a un nivel de 5,1 a 13,1 para papilomas solitarios y 4,4-7,0 para micro-papilomas. Claramente, la presencia de atipia y el número de lesiones desempeñan un papel en la determinación del futuro riesgo de cáncer de mama (KILUK, 2007).

La hiperplasia ductal florida o moderada representó el $9,19 \%$ de todas las lesiones benignas de mama, siendo la lesión proliferativa sin atipia más frecuente, comparado con el $12.5 \%, 10 \%, 22 \%$ documentado en Detroit, Tokio y New York respectivamente (SCHUERCH et al., 1982; DEVITT, 1986) tiene un pico de incidencia en el rango de edad de los $35-55$ años con un $56.25 \%$, con una edad media de 44 años, lo que concuerda con lo documentado por (TAVASSOLI y NORRIS, 1990), donde observaron una edad media de 45 años y una frecuencia en el rango de edad de 31-45 años del $43 \%$ y en mayores de 45 años del $44 \%$.

De las lesiones proliferativas con atipia, la hiperplasia ductal atípica es la más frecuente con el $2,84 \%$, seguida por la hiperplasia lobulillar atípica con 1 solo reporte que representa el $0,22 \%$ de todos los casos, cifras muy cercanas a los resultados de un estudio (TICE et al., 2013), donde la hiperplasia ductal es la lesión proliferativa con atipia más frecuente con el 2,9\% de los casos de enfermedad benigna de mama, seguido de la hiperplasia lobulillar atípica con el 0,7\% (TICE et al., 2013). Son notablemente más frecuentes en mujeres entre de $35-55(46,6 \%)$ y mujeres mayores de 55 años (40\%), con una edad promedio de 53 años. Resultados de un estudio realizado en EEUU confirman un RR de cuatro veces mayor de cáncer de mama posteriormente en mujeres con hiperplasia atípica. Una historia familiar positiva no confiere riesgo significativamente mayor en las mujeres con atipia. Las mujeres con atipia diagnosticadas en edades más tempranas tienen un RR mayor en comparación con las tasas de edad esperados. EI RR es de 6,76 a la edad de menos de 45 años, 5,10 a los 45 a 55 años, y 2,87 a la edad mayor de 55 años (DEGNIM et al., 2007).

La importancia de la hiperplasia atípica como marcador biológico para el aumento del riesgo de desarrollar cáncer de mama invasivo se ha confirmado en un estudio multicéntrico que incluyó a más de 280.000 mujeres (DUPONT y PAGE, 1985; DUPONT et al., 1993).

\section{Conclusiones}

La enfermedad benigna de la mama amerita una evaluación patológica precisa y se debe tener un patrón o protocolo de clasificación con criterios morfológicos que permita pronosticar el riesgo de cáncer personal de la paciente. La clasificación en lesión benigna proliferativa o no proliferativa basada en los estudios de Page y Dupont (1985) es de fácil aplicabilidad. En la reevaluación de los casos se mejoró la exactitud diagnóstica y la valoración del riesgo de cáncer. De las lesiones benignas de la mama estudiadas en el periodo 2012-2013 en el Departamento de Sucre las de tipo no proliferativo son las más comunes con una prevalencia del $82,27 \%$.

\section{Referencias}

BAFAKEER, S.S.; BANAFA, N.S.; ARAM, F.O. 2010. Breast diseases in Southern Yemen. Saudi Med J. (9):1011-4.

BOJANINI, J.F.; VILLEGAS, J.C. 1993. Características clínicas de la Enfermedad Benigna de la mama. Hospital General de Medellín Luz Castro de Gutiérrez. Revista CES Medicina (8):51-62. 
CHENG, J.; QIU, S.; RAJU, U,; WOLMAN, S,R.; WORSHAM, M,J. 2008. Benign breast disease heterogeneity: association with histopathology, age, and ethnicity. Breast Cancer Res Treat. (111):289-296.

CIATTO, S,; BONARDI, R.; RAVAIOLI, A.; CANUTI, D.; FOGLIETTA, F.; MODENA, S. 1998. Benign breast disease surgical biopsies, are they always justified? Tumori (5):521-524.

DEGNIM, A.C.; VISSCHER, D.W.; BERMAN, H.K.; FROST, M.H.; SELLERS, T.A.; VIERKANT, R.A. 2007. Stratification of breast cancer risk in women with atypia: a Mayo cohort study. J Clin Oncol (25):2671-7.

DEVITT, J.E. 1986. Benign disorders of the breast in older women. Surg Gynecol Obstet (162):340-2.

DONEGAN, W.L.; SPRATT, J.S. 1995. Cancer of the breast. British Journal of Surgery (9):1292.

DUPONT, W.D.; PAGE, D.L.; PARL, F.F.; VNENCAK-JONES, C,L.; PLUMMER, W.D.; RADOS, M.S.; SCHUYLER, P.A. 1994. Long-term risk of breast cancer in women with fibroadenoma. N Engl J Med. (331):10-15.

DUPONT, W.D.; PAGE, D.L. 1985. Risk factors for breast cancer in women with proliferative breast disease. N Engl J Med. (312):146-151.

EL-WAKEEL, H.; UMPLEBY, H.C. 2003. Systematic review of fibroadenoma as a risk factor for breast cancer. The Breast (12):302-307.

FITZGIBBONS, P.L.; HENSON, D.E.; HUTTER, R.V. 1998. Benign breast changes and the risk for subsequent breast cancer: an update of the 1985 consensus statement. Cancer Committee of the College of American Pathologists. Arch Pathol Lab Med. (122):1053-1055.

GOEHRING, C.; MORABIA, A. 1997. Epidemiology of benign breast disease, with special attention to histologic types. Epidemiol Rev. (19):310-327.

GOMERA, D.R.; BERROA, K.C.; TEJEDA, M.C.; ALCÁNTARA, M,; PICHARDO, Y.; MEJÍA, J.F. 2000. Patologías benignas de la mama: frecuencia diagnóstica en Laboratorio Nacional Dr. Defilló. Rev Med Dom (61):40-43.

HUGHES, L.E.; MANSEL, R.E.; WEBSTER, D.J.T. 1987. Aberration of normal development and involution (ANDI): a new perspective on pathogenesis and nomenclature of benign breast disorders. The Lancet (330):1316-19.

JABBO, N.S.; JASSIM, H.A. 2010. Pattern of Benign Female Breast Disease in Al-Yarmouk Teaching Hospital. Mustansiriya Medical Journal (9):21-24.

KILUK, J.V.; ACS, G.; HOOVER, S.J. 2007. High-Risk Benign Breast Lesions: Current Strategies in Management. Cancer Control (4):321-329.

MARREROS, J.E.; CONTRERAS, H.M.; GARCÍA, L. 2013. Benign breast pathology in patients attended at Hospital Nacional Arzobispo Loayza Gynecology Oncology service. Rev. Peru Ginecol Obstet. (59):161-165.

MEMON, A.; PARVEEN, S.; SANGRARASI, A.K.; MALIK, A.M.; LAGHARI, A. 2007. Changing pattern of benign breast lumps in young females. World J Med Sci. (2):21-4.

MERIH, G.; AYSEGUL, A. 2006. Benign Breast Diseases: Classification, Diagnosis and Management. The Oncologist (11):435-449.

OCHICHA, O.; EDINO, S.T.; MOHAMMED, A.Z.; AMIN, S.N. 2002. Benign breast lesions in Kano. Nigerian Journal of Surgical Research (1):1-5.

OLU-EDDO, A.; UGIAGBE, E.E. 2011. Benign breast lesions in an African population: A 25-year histopathological review of 1864 cases. Nigerian Medical Journal (4):211.

SCHNITT, S.J.; JIMI, A.; KOJIRO, M. 1993. The increasing prevalence of benign proliferative breast lesions in Japanese women Cancer (8):2528-2531. 
SCHUERCH, C.; ROSEN, P.P.; HIROTA, T.; ITABASHI, M.; YAMAMOTO, H.; KINNE, D.W.; BEATTIE, E.J. 1982. A pathologic study of benign breast diseases in Tokyo and New York. Cancer (50):1899-1903.

SHIRLEY, S.E.; MITCHELL, D.; SOARES, D.P.; JAMES, M.; ESCOFFERY, C.T.; HODEN, A.M.; WOLFF, C.; CHOY, J.; WILKS, J. 2008. Clinicopathologic Features of Breast Disease in Jamaica: Findings of The Jamaican Breast Disease Study, 2000-2002. West Indian Med J. (2):90.

SINGLETARY, S,E. Rating the risk factors for breast cancer. Ann Surg. 2003. (237):474-482.

TAVASSOLI, F.A.; NORRIS, H.J. 1990. A comparison of the results of long-term follow-up for atypical intraductal hyperplasia and intraductal hyperplasia of the breast. Cancer (65):518-529.

TICE, J.A.; O'MEAR, E.S.; WEAVER, D.L.; VACHON, C.; BALLARD-BARBASH, R.; KERLIKOWSKE, K. 2013. Benign Breast Disease, Mammographic Breast Density, and the Risk of Breast Cancer. Journal National Cancer Inst. (14):1043-1049.

VALDIVIESO, R.; LIMÁS, M.; ALVINES, H. 1999. Lesiones benignas de la mama. Ginecol Obstet Perú (1): 69-72.

WANG, J.; COSTANTINO, J.P.; TAN-CHIU, E.; WICKERHAM, D.L.; PAIK, S.; WOLMARK, N. 2004. Lower-category benign breast disease and the risk of invasive breast cancer. Journal National Cancer Inst. (96):616-620.

WEIDNER, N.; BREAST, I.N.; WEIDNER; COTE; SUSTER; WEISS. 2003. Modern Surgical Pathology. Saunders. Philadelphia, USA.

WORSHAM, M.J.; ABRAMS, J.; RAJU, U.; KAPKE, A.; LU, M.; CHENG, J.; MOTT, D.; WOLMAN, S.R. 2007. Breast cancer incidence in a cohort of women with benign breast disease from a multiethnic, primary health care population. The Breast Journal (13):115-121. 